\title{
A study of the suitability of disposable coloured contact lenses for a South African clinic based pop- ulation
}

\section{VR Moodley*}

\author{
Discipline of Optometry, University of KwaZulu-Natal, Westville Campus, Private Bag X54001, \\ Durban, 4000 South Africa \\ <moodleyvr@ukzn.ac.za>
}

Received 28 August 2009; revised version accepted 27 October 2009

\section{Abstract}

Optometrists have always considered the fitting of all types of contact lenses to be an integral part of the scope of practice of their profession. However, since the introduction of disposable cosmetic coloured contact lenses into the market, the frequency of over the counter sales of contact lenses has significantly increased. Patients often present with contact lens complications at the University of Kwa-Zulu Natal clinic and local practices. It is sometimes reported that the lenses were purchased over the counter, from an optometry practice or another non-registered vendor. A common observation in these instances has been that the lenses do not fit optimally and that the incidence of complications associated with tight fitting lenses was much higher amongst the African patients as opposed to those of other race groups.

A clinical observational study utilizing convenience sampling of 240 subjects was undertaken to evaluate the fitting criteria of disposable cosmetic coloured contact lenses on a South African clinic based population. All patients requesting the lenses chose their preferred colour and were fitted with Freshlook ColorBlends, Images or Expressions Colors lenses. Lenses were evaluated after 20-25 minutes and then classified into tight, ideal or loose fits according to the lower lid push-up test, lens centration, post blink movement and version lag
\end{abstract}

and upgaze lens movements.

The ages of the subjects ranged from 16 to 45 years with a mean of $24.13 \pm 5.66$ years. Seventy $(29.2 \%)$ were males and $170(70.8 \%)$ were females. The majority $(62.9 \%)$ of the lenses fitted on the subjects were rejected according to the fit criteria. The African subjects had the highest percentage of rejected fits $(82.8 \%)$ whilst $75 \%$ of the White subjects had acceptable fits. The main reason for lens fits being rejected was that they displayed characteristics of a tight fit ( $96 \%$ ) with only $4 \%$ of the fits being rejected due to being too loose. These results highlight the need for contact lens manufacturing companies to realize that those lenses that they design and which undergo clinical trials on Caucasian eyes do not necessarily fit well on the majority of patients in this part of the world. The reasons for this may be related to differences in corneal profiles of the different population groups with some being more suitable to particular lens designs than others. It is recommended that the public and practitioners be educated about the potential dangers of over the counter sales of contact lenses and that pre-fitting evaluations consultations of all contact lenses are an essential part of contact lens care.

Key words: Disposable contact lenses, cosmetic coloured lenses, over the counter (OTC) sales, contact lenses and South Africa 


\section{Introduction}

The scope of practice of an optometrist in South Africa is defined as per the 1974 Regulation $^{1}$ as:

a) the performance of eye examinations on patients with the purpose of detecting visual errors in order to provide clear, comfortable and effective vision; and

b) the correction of errors of refraction and related factors by the provision of spectacles, spectacle lenses and contact lenses, and the maintenance thereof, and the use of scheduled substances as approved by the board and the Medicine Control Council or by any means other than surgical procedures.

The introduction of cosmetic coloured contact lenses into the market has created numerous unsafe practices associated specifically with these lenses. One of the common practices that raise concerns amongst the optometric profession in South Africa is the increasing number of contact lenses being bought over the counter from flea markets, trade fairs, clothing stores, beauty shops and through the internet without going through the proper clinical fitting regimen. In the past two years, many unregistered people began selling cosmetic coloured contact lenses within the country and had to be challenged legally by the profession in an attempt to stop this practice. Their response to the challenge was that the regulation, as it is phrased, implies that the dispensing of contact lenses for the correction of refractive errors only was within the scope of practice of the optometrist and not the supply of contact lenses for non-refractive or cosmetic purposes. The difficulty thus facing prosecuting authorities arose from this claim that the plano coloured contact lenses are merely for cosmetic enhancement and hence the non-registered vendors were not acting illegally as they were not dispensing the lenses to correct refractive errors. This issue had additionally concerned the regulator, the Health Professions Council of South Africa (HPCSA), a statutory body whose mandate it is to protect the public. After much debate and investigation into the issue, the wording of the regulation governing the scope of the profession of optometry had to be reviewed and the HPCSA proposed to the government an amendment to the regulation to include "the supply and fitting of any contact lenses to members of the public" to be within the scope of the Optometric profession (Government Notice No.R280 of 5 April 2007) $)^{2}$. The supply and fitting of all types of contact lenses will thus form part of the scope of practice of optometrists and will be fully regulated in South Africa. The amended regulation translates to mean that it is illegal for any non-registered person to fit or supply contact lenses directly to the public and it meets the necessary legal requirements for appropriate action to be taken against anyone contravening the Act. This right of optometrists to fit contact lenses on patients is accompanied by the responsibility to ensure that patient care remains paramount at all times in this process.

A contact lens rests on the cornea and interacts with the ocular structures whilst it is being used. The assessment of a soft contact lens fit by observation of the lens behaviour on the eye is one of the most common procedures undertaken in contact lens practice ${ }^{3}$. In fitting contact lenses, procedures are routinely conducted by the practitioner to determine both the patient's suitability for wearing contact lenses and which particular lens type will be the most suitable lens to be worn by the pre-selected patient. The practitioner also has to have the necessary equipment to fit lenses as it is not possible, for instance, to fit contact lenses or provide ongoing care for the patient without proper access to a slit-lamp biomicroscope ${ }^{4}$. The value of the consultation has been emphasized by authors ${ }^{5,6}$ who state that the first stage of contact lens fitting is a comprehensive examination to determine the advisability of application and the probability of success with each patient as well as to educate the patient about contact lens wear. Patients who are contraindicated to contact lens wear should, at this first stage, be informed about their unsuitability for lens wear. The contact lens design with appropriate parameters must be selected by the optometrist as a final lens for successful wear to be achieved. The contact lens dispensing is then followed by scheduled aftercare consultations to evaluate the lenses and manage any problems which may have arisen with wear. For safe and successful wear, it is critical that the lens fitted does not compromise the integrity of the ocular structures during wear. The United States Federal Fairness to Contact Lens Consumers Act (FCLCA) ${ }^{7}$, which took effect in February 2004, defines a contact lens fitting as, "the process that begins after the initial eye examination and ends 
when a successful fit has been achieved or, in the case of a renewal prescription, ends when the prescriber determines that no change in prescription is required, and such term may include: A) an examination to determine lens specifications; B) except in the case of a renewal of a prescription, an initial evaluation of the fit of the lens on the eye; and C) medically necessary follow-up examination".

Problems associated with contact lens wear may affect the eyelid, the conjunctiva, the various layers of the cornea, and even the tear film that covers the outer surface of the eye $\mathrm{e}^{8,9}$. Stapleton et al ${ }^{10}$ found that the risk of eye disease associated with internet purchases of contact lenses is almost five times more than when lenses were bought from an optometrist. Individuals who purchase over the counter contact lenses frequently complain of painful, red eyes, poor vision or other contact lens related problems. On investigation by the practitioner, it is often found that the lenses are unsuitable for the patient as a result of the material or design being inappropriate, the lens being poorly maintained by the patient or either too tight or loose fitting for the specific corneal profile. A study ${ }^{11}$ of the influence of lens design on clinical performance concluded that the back surface design of a contact lens has a significant effect on lens fit. Young and Coleman ${ }^{12}$ found in their study that poorly fitting lenses affect ocular integrity and that corneal staining and other physiological effects of soft contact lens wear vary with the quality of lens fit. Reports given by patients who were examined at the University of Kwa Zulu-Natal (UKZ-N) clinic indicate that, on certain occasions, the ill-fitting lenses have been supplied over the counter in optometry practices without the appropriate clinical consultation having been done. This practice, apart from being extremely unsafe for the patient, is also unethical and illegal and in violation of the HPCSA regulations governing the clinical practice of optometry.

The author and other clinical faculty have over the years noticed an increasing number of patients who presented at the UKZ-N clinic or at private practice with contact lens complications arising from poorly fitted cosmetic coloured contact lenses. An additional observation is that the incidence of complications associated with tight fitting lenses was much higher amongst the African patients as opposed to those of other racial groups. The general perception that cos- metic contact lenses are merely for cosmetic enhancement and serve a similar function as eye make-up such as mascara, eye liners or artificial eyelashes has contributed to the difficulty in getting the public to accept that these lenses are regulated medical devices that need to be fitted by a registered professional. This perception has also resulted in patients believing that lenses can be chosen on the basis of colour only and will be suitable on all eyes. Patients therefore decide to purchase lenses through any available source or present at optometry practices expecting to buy the lenses over the counter.

These observations of a perceived 'one-size fits all' notion by the public and the resulting unsafe practices that occur had led the author to conduct an evaluation of the fitting characteristics of locally available cosmetic coloured contact lenses on patients presenting at the research sites requesting to be fitted with these lenses. The objective of the investigation was to determine whether these lenses being purchased over the counter meet the acceptable fitting characteristics ${ }^{3,13-16}$ that are commonly used by practitioners for soft contact lens assessments in the majority of the population.

\section{Methodology}

The patients were selected from both the UKZ-N optometry clinic and a local private practice. A clinical observational study design using convenience sampling for patient selection was used. All patients presenting and requesting cosmetic contact lenses were included in the investigation. Two hundred and forty patients (480 eyes) were fitted with coloured contact lenses. In an attempt to replicate the method of choosing lenses that occurs with over the counter sales, the lenses were selected according to the patient's preference of a particular colour. Although not analysed for the purposes of this study, standard prefitting procedures used when fitting contact lenses such as refraction, slit lamp evaluation, keratometry, tear function tests and other relevant ocular measurements were conducted on each subject for subsequent patient care. The specific brand of contact lenses was selected according to the approximate trial lens power availability and patient preference when provided with the choice of colours in the various brands. Patients could choose from three available 
brands namely Images ${ }^{\circledR}$, Freshlook ColorBlends ${ }^{\circledR}$ or Expressions Colors ${ }^{\circledR}$ (previously Frequency Colors) lenses. These particular lenses were used in the study due to their availability as trial contact lenses at the respective study sites. The parameters of the lenses are indicated in Table 1.

Table 1: The base curves, diameters, design and Dk/t values of the lenses used in this study. All the lenses used have low Dk/t values.

\begin{tabular}{|l|c|c|c|c|}
\hline \multicolumn{1}{|c|}{ LENS TYPE } & $\begin{array}{c}\text { BASE } \\
\text { CURVE }\end{array}$ & DIAMETER & DESIGN & $\begin{array}{c}\text { Dk/t } \\
(-3.00 \mathrm{DS})\end{array}$ \\
\hline Freshlook ColorBlends & $8.6 \mathrm{~mm}$ & $14.5 \mathrm{~mm}$ & Spherical & 20 \\
\hline Expressions Colors & $8.7 \mathrm{~mm}$ & $14.4 \mathrm{~mm}$ & Aspheric & 19 \\
\hline Images & $8.6 \mathrm{~mm}$ & $14.2 \mathrm{~mm}$ & Spherical & 17 \\
\hline
\end{tabular}

The trial lenses clinical assessment involved the subject wearing the lenses for 20-25 minutes before the lenses were assessed for fit characteristics. Lens fit characteristics were assessed and classified as ideal, tight or loose according to commonly used norms for the various dynamic and static soft lens fitting criteria $^{13-16}$. Lenses were then either accepted or rejected according to the results of the lower lid push-up test, lens centration, post-blink movement and version lag and upgaze lens movements. Only lenses that met the criteria for an ideal contact lens fit were accepted and lenses not meeting these criteria were rejected on the basis of being either too tight or loose fitting ${ }^{13-16}$. Visual acuity and subjective response were not considered as factors for acceptance or rejection of the lenses. Young ${ }^{12}$ found that subjective comfort was of little value in identifying tight fitting lenses but was of some limited value in identifying loose fitting lenses. Patients who were not successfully fitted with the study lenses were subsequently provided with the option of being fitted with other suitable lenses on the market to ensure overall patient care was not compromised. Data was captured and analyzed using Microsoft Excel.

\section{Results}

There were 240 subjects in the study of which 70 $(29.2 \%)$ were male and $170(70.8 \%)$ were female. The ages ranged from 16 to 45 years with a mean age of $24.14 \pm 5.66$ years. The racial profile of the subjects is as shown in Figure 1.

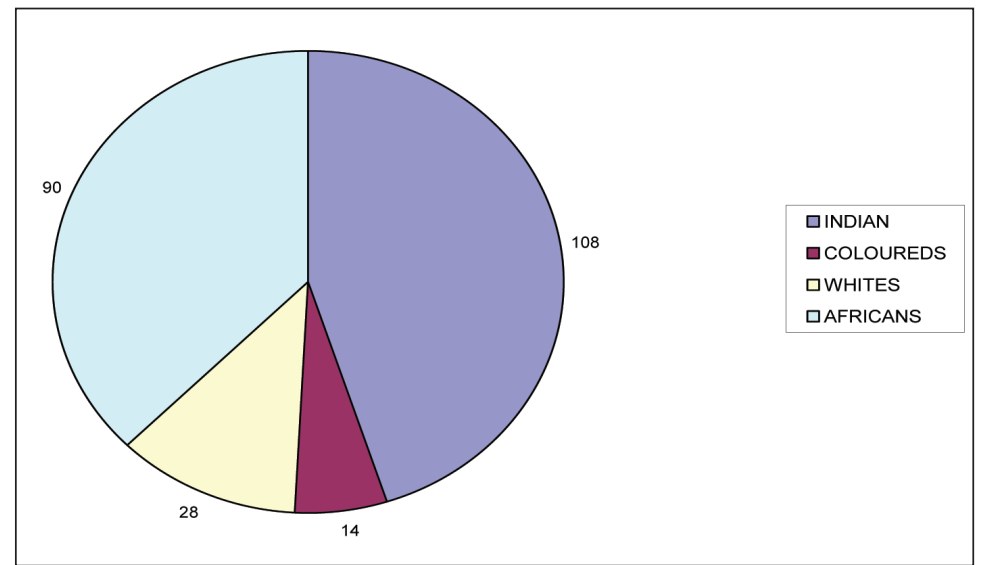

Figure 1: Diagram showing the number of subjects in each race group $(N=240)$ who participated in the study. Most of the subjects were African or Indian.

Each patient was allowed to select lenses which they preferred according to colour choice and a trial lens closest to the subjects refractive power was then fitted. Due to the larger range of powers available in Expressions Colors ${ }^{\circledR}$, most of the patients (77.5\%) chose these lenses whilst $17.1 \%$ were fitted with Freshlook ColorBlends ${ }^{\circledR}$ and $5.4 \%$ with Images ${ }^{\circledR}$.

It was found that the majority $(62.9 \%)$ of the subjects could not be fitted with the selected cosmetic coloured contact lenses and only $37.1 \%$ had ideal fits. The African subjects had the smallest number of ideal fits (20\%) amongst the groups whilst $75 \%$ of the White subjects had achieved acceptable fits with the contact lenses fitted as shown in Figure 2.

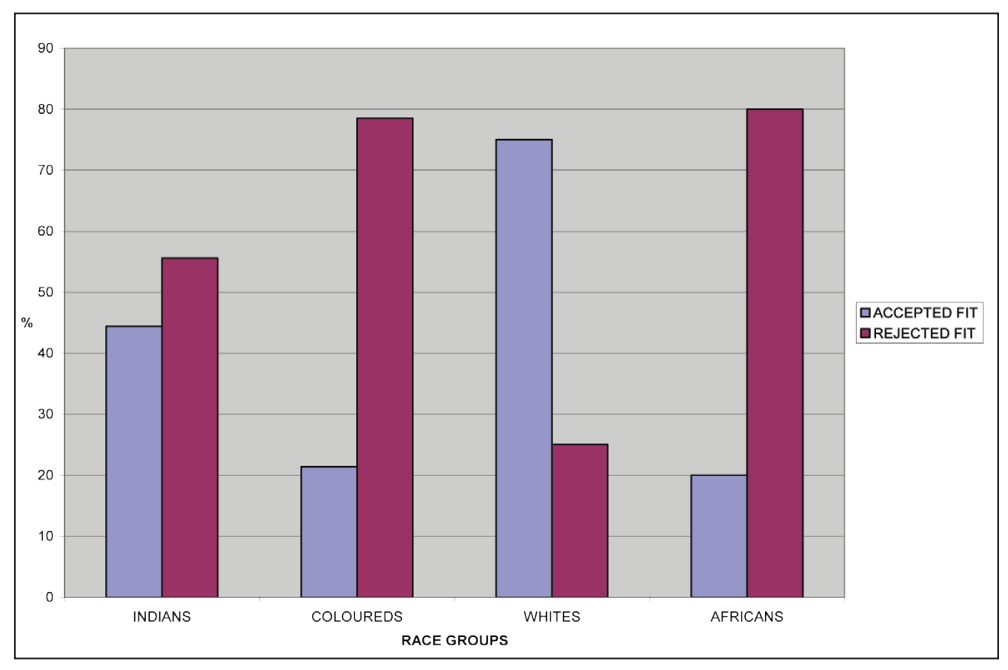

Figure 2: Diagram showing the percentage of accepted and rejected contact lens fits for each race group. 
Table 2: Table showing the percentages of accepted and rejected fits for each race group according to lens type. The percentage rejection was highest among the African and Coloured subjects.

\begin{tabular}{|c|c|c|c|c|c|c|c|c|c|c|c|c|}
\hline \multirow{3}{*}{ LENS } & \multicolumn{3}{|c|}{ AFRICANS } & \multicolumn{3}{|c|}{$\begin{array}{l}\text { INDIANS } \\
\end{array}$} & \multicolumn{3}{|c|}{ COLOUREDS } & \multicolumn{3}{|c|}{ WHITES } \\
\hline & \multirow{2}{*}{$\frac{\text { ACCEPT }}{\text { Ideal }}$} & \multicolumn{2}{|c|}{ REJECT } & \multirow{2}{*}{$\frac{\text { ACCEPT }}{\text { Ideal }}$} & \multicolumn{2}{|c|}{ REJECTED } & \multirow{2}{*}{$\frac{\text { ACCEPT }}{\text { Ideal }}$} & \multicolumn{2}{|c|}{ REJECTED } & \multirow{2}{*}{$\frac{\text { ACCEPT }}{\text { Ideal }}$} & \multicolumn{2}{|c|}{ REJECTED } \\
\hline & & Tight & Loose & & Tight & Loose & & Tight & Loose & & Tight & Loose \\
\hline EXP & 17.8 & 60.0 & 3.3 & 38.9 & 40.7 & 2.8 & 14.3 & 57.1 & 0.0 & 39.3 & 10.7 & 0.0 \\
\hline FLK & 2.2 & 12.2 & 0.0 & 3.7 & 10.2 & 0.0 & 0.0 & 14.3 & 0.0 & 25.0 & 14.3 & 0.0 \\
\hline IM & 0.0 & 4.4 & 0.0 & 1.8 & 1.9 & 0.0 & 7.1 & 7.1 & 0.0 & 10.7 & 0.0 & 0.0 \\
\hline TOTAL & 20.0 & 76.6 & 3.3 & 44.4 & 52.8 & 2.8 & 21.4 & 78.5 & 0.0 & 75.0 & 25.0 & 0.0 \\
\hline
\end{tabular}

Tight fits accounted for $96 \%$ of the unsuccessful fits and loose fits only 4\%. The lenses were too tight for the majority of the African, Coloured and Indian patients as shown in Table 3. Chi square analysis revealed that the rejection of the lenses was influenced by the type of lenses used with the African subjects having the highest rejection of all brands of these cosmetic coloured lenses $(p=0.040)$ and the White subjects the lowest $(p=0.49)$.

\section{Discussion}

The concerning trend currently practiced with coloured contact lenses wear involves the patient being given lenses over the counter purely on requesting for them, without any pre-trial fitting procedures being performed. This practice generally excludes any clinical examination to ascertain the suitability of the patient for contact lens wear or the determination of appropriate lens type, design or parameters for the patient. Hence the methodology used in this study, involving lenses being fitted purely on patient request for cosmetic lenses, was chosen in order to mimic the current practice. If all potential contact lens wearers around the world had the same medical and ocular health conditions, anatomy, physiology and identical corneal profiles this practice may be deemed to be relatively safe. However, this is not the case as shown in a study by Wong et $a{ }^{17}{ }^{17}$ who found that due to the steeper corneas of the Chinese eyes, about $40 \%$ of their subjects were unable to obtain acceptable fits with a particular one-fit frequent replacement lens. Lenses with different parameters will need to be available in the lens evaluated to allow more people in that population to wear the brand. The authors further stated that, as there are individual variations of lens fitting behaviour, trial lens fitting assessment is the most suitable way to select the appropriate lens. These results are supported by other studies ${ }^{18-21}$ which indicate that there are ethnic differences which cause differences in ocular characteristics such as horizontal visible iris diameter (HVID), corneal radius, palpebral aperture or tear break up time (TBUT). Noting these varying anatomical and physiological characteristics of different ethnic groups, as well as differences in age and gender, it would therefore be expected that lenses may fit differently on different groups of people ${ }^{22}$. The results of this study show that the cosmetic coloured contact lenses tried did not meet the acceptable fitting criteria ${ }^{13-16}$ and were rejected by the consulting clinicians for the majority of subjects who presented for them. Ninety six percent of the lenses that were rejected due to their poor fitting characteristics were rejected due to tight fits. This could be due to the fact that all the lenses tried were only available in one design, base curve and diameter as shown in Table 1. Young et al., in a study to evaluate the fit and clinical behaviour of eight disposable hydrogel contact lenses, found that although all the lenses were designed to fit a wide variety of eyes, they exhibited widely varying fitting characteristics with some being more successful than others ${ }^{23}$. The fact that, in this study, the lenses were too tight for the majority of the non-White subjects supports previous researchers' claims of ethnic variations in ocular characteristics ${ }^{17-21}$. It could also be explained by the observations made by clinicians at the university clinic and anecdotal reports from numerous local optometrists that the African and Coloured patients, in particular, appear to have flatter corneas than their White counterparts. The number of Coloured subjects participating in the study, as compared with the other non-white groups, was relatively small and although the results revealed mostly tight fits, a larger sample 
size will be advised for further studies in this area.

In order to have lenses available for the large number of subjects that could not be fitted in this study, companies could consider changing the materials, manufacturing method, increasing the range of base curves or diameters offered to alter the sag of the lenses or altering the lens designs to create lens fits that meet acceptable fitting criteria for the African population. Maldonado and Efron ${ }^{24}$ have shown that manufacturing method and material composition have a fundamental effect on the fitting characteristics of a lens. Manufacturers usually conduct clinical trials with their lenses and decide on lens parameters that will fit the majority of the population. The manufacturers of the lenses used in this study are multinational corporations and their lenses are usually designed and distributed after being clinically evaluated in certain countries only. Most often clinical contact lens trials are conducted in the United States of America (USA), Europe and Australia on mainly Caucasian eyes. Presumably, none of the lenses used in this study have been clinically tried on subjects from Africa to attempt to ascertain their suitability for the subjects from this part of the world. This might have contributed to the reason why the lens parameters were inappropriate for the majority of the subjects in this study with the primary reason for lens rejection being that the lenses were too tight. However, as seen in Figure 2, the majority of the White patients, who were of European decent, were mostly successfully fitted with these lenses. This suggested that they may have similar corneal profiles to those of the Caucasian subjects that were used in the clinical trials of these lenses.

The majority of patients wearing contact lenses over the years in South Africa have been White patients, who achieved good fits with the available disposable cosmetic lenses in this study. This may explain the limited range of parameters that companies have available as the need for other parameters more suitable to other ethnic groups may not have been previously realized. However, the African population in South Africa has made major inroads into the economic arena since 1994 and, with the introduction of affirmative action policies, they now have access to higher paying jobs and many more entrepreneurial business opportunities. Many African patients are now able to afford contact lenses and are increas- ingly requesting this mode of correction as their first choice of correction. It is thus imperative, from a public health perspective, for the contact lens industry to ensure that they offer lenses that are designed to fit properly and cause minimal risk to the ocular health of this category of patients. Without properly designed disposable lenses being available for the increasing number of non White, and in particular African patients requesting cosmetic disposable contact lenses, optometrists will realize that this deficiency results in compromised ocular health to their patients and a significant loss of income to their practices.

It is noteworthy that optometrists who fit these lenses that compromise ocular integrity, merely for the financial gain are practicing unethically, being clinically negligent and are guilty of unprofessional conduct. In the US about $40-50 \%$ of all malpractice claims involving optometrists arise from the fitting or wear of contact lenses ${ }^{25}$. Optometrists must take full responsibility for their patient care at all times and lenses should not be dispensed if they do not fit properly, even if the patient's needs are purely cosmetic. In particular, optometrists must ensure that lenses are never provided over the counter in their practices by any employee without the practitioner's permission as the practitioner is vicariously liable for the actions of any of his or her employees in the practice that causes harm to a patient ${ }^{26}$. The fact that the majority of the subjects in the present study were unable to be successfully fitted with the lenses makes it necessary for pre-trial examinations to be conducted prior to contact lenses being dispensed to patients and patients must also be educated about the dangers of wearing lenses that have not been fitted and prescribed by a registered optometrist. The clinical consultation allows for the optometrist to educate the patient on the lens care and maintenance regimen as well as the importance of patient compliance. Improvements in the level of patient compliance with instructions, is likely to bring about increased patient success with contact lens wear ${ }^{27}$.

This study also highlights the risk that South African patients purchasing cosmetic lenses over the counter face as the lenses may, in the majority of cases, not fit the patient properly. As most of the lenses in this study showed fitting characteristics of tight lenses, the patients will initially be unaware of the problems experienced as experience has shown that 
the tendency is for tight lenses to initially feel more comfortable than an ideal fit or a loose lens. This may result in the patient believing that the lens fit is good as it is "hardly felt" and yet eventually results in complications such as corneal staining, conjunctival staining corresponding to lens edge ${ }^{16}$, perilimbal vascularization or other long term corneal changes such as chronic infections. The tight fit, compounded by the fact that all these lenses have a low $\mathrm{Dk} / \mathrm{t}$ value, may additionally expose the patient to hypoxia-related contact lens complications. In a study to illustrate the implications of the unauthorized sale and unmonitored wearing of decorative cosmetic contact lenses resulting in ocular complications, Steineman et al28 concluded that uninformed lens wearers experienced acute vision-threatening infections and inflammation. Other studies ${ }^{29,30}$ have also highlighted the potential ocular complications of wearing cosmetic coloured contact lenses bought from unlicensed vendors.

The clinical care and welfare of patients has to remain central to all those involved in the manufacture, supply and fitting of contact lenses. The evidence provided in this study, that is, that currently most of the disposable cosmetic contact lenses do not meet the optimal fitting criteria for certain categories of patients in South Africa makes obtaining of lenses from sources other than registered practitioners particularly unsafe for patients. It is important that contact lens wear is always associated with optimum contact lens care. This contact lens care must always include the initial examination, proper patient education on lens care and management with regular follow up care.

\section{Recommendations}

It is recommended that contact lens manufacturers realize that in order to adequately service the South African population, the majority $(79.3 \%$ according to the most recent estimated national population census) $)^{31}$ of whom are African, they will need to invest in research and development of contact lenses that fit the flatter corneal profile and minimize the risk of any adverse corneal compromise. South Africans could be included in the clinical trials, as the results of this study suggest that perhaps contact lenses that have undergone clinical trials in the US, Australia or Europe alone do not meet the fitting parameters required in this part of the world. The inclusion of more Af- ricans in clinical trials will also have the potential to increase the number of contact lens wearers in South Africa. Optometrists must refrain from providing patients with lenses without the appropriate clinical protocols being followed to ensure suitability of lenses provided and illegal vendors of contact lenses must be reported to the appropriate law enforcement agency for necessary action to be taken against them. Future studies to investigate further the possible differences in ocular characteristics between the various race groups in South Africa is recommended to contribute to the design and technological developments of contact lenses.

\section{Conclusion}

Acknowledging possible limitations of a relatively small sample size, this study reveals that current available coloured contact lenses in South Africa do not meet the design, parameter and fitting characteristics to provide optimal fits for a large proportion of the population. These lenses thus have the potential to cause ocular complications to an unsuited wearer and should therefore only be prescribed following comprehensive lens fitting procedures. In the interest of good public health, over the counter sales of these lenses should be discontinued instantly. Eye care professionals have an ethical and legal obligation to ensure that they too refrain from any unsafe practices associated with contact lenses and the public must continually be educated on safe practices for the use of contact lenses, irrespective of the type and design of the lens. Regulatory authorities in South Africa should refine the regulations governing the supply and fitting of contact lenses to ensure patient comfort and safety, which must at all times, remain central to our overall professional responsibilities.

\section{References}

1. South African National Health Professions Act 56 of 1974. Regulation 2 (1) of Act No. 56 of 1974.

2. South African National Department of Health Government Notice No.R.280 of 5 April 2007.

3. Young G. Evaluation of Contact Lens Fitting Characteristics. Optom Vis Sci 1996 73(4) 247-254.

4. Goldberg JB. Biomicroscopy for Contact Lens Practice: Clinical Procedures. Chicago: Professional Press 1970.

5. White P, Barr J. Preventative contact lens care. Contact Lens Spectrum 2001 http://www.clspectrum.com/article. aspx ?article=11933 (last accessed 25/10/09). 
6. Remba MJ. In: Mandel RB. Contact Lens Practice, 4th Edition. Springfield IL: Thomas 1988 p136.

7. Seibel DB. Fairness to Contact Lens Consumers Act: The Final Rule Optometry 200475 (8) 527-534.

8. Efron N. Contact Lens Complications, 2nd Edition. Oxford: Butterworth-Heinemann 2008.

9. Radford CF, Minassian D, Dart JKG, Stapleton F, Verm S. Risk factors for nonulcerative contact lens complications. Ophthalmol 2009116 (3) 385-392.

10. Stapleton F, Keay L, Edwards K, Naduvilath T, Dart JKG, Brian G, Holden BA. The incidence of contact lens-related microbial keratitis in Australia. Ophthalmol 2008115 (10) 1655-1662.

11. Young G, Holden B, Cooke G. Influence of soft contact lens design on clinical performance. Optom Vis Sci 199270 (5) 394-403.

12. Young $\mathrm{G}$, Coleman S. Poorly fitting soft lenses affect ocular integrity CLAO J 200127 (2) 68-74.

13. Vey J, Meyler J, Davies J. Essential Contact Lens Practice. Oxford: Butterworth Heinemann 2002.

14. Mandell RB. Contact Lens Practice, 4th edition. Springfield: IL Thomas 1989 544-550.

15. Gasson A. Soft lens fitting. In: Phillips AJ, Stone J. Contact Lenses, 3rd edition. London: Butterworth, 1989 400-417.

16. Sulley A. Contact lens fitting today Part 2: Soft contact lens fitting. Optometry Today 2005 pp 46-51.

17. Wong MK, Lee TT, Poon MT, Cho P. Clinical performance and factors affecting the physical fit of a soft toric frequent replacement contact lens. Clin Exp Optom 200285 (6) 3507.

18. Lam CSY, Loran DFC. Designing contact lenses for Oriental eyes. Cont Lens Ant Eye 199114 109-114.

19. Matsuda LM, Woldorff CL, Kame RT, Hayashida JK. Clinical comparison of corneal diameter and curvature in Asian eyes with those of Caucasian eyes. Optom Vis Sci 199269 51-54.

20. Patel S, Virhia SK, Farrell P. Stability of the precorneal tear film in Chinese, African, Indian and Causian eyes. Optom Vis Sci 199572 911-915.

21. Cho P. Reliability of a portable noninvasive tear break up time test on Hong Kong Chinese. Optom Vis Sci 199370 1049-1054.

22. Wong MK, Goto T, Klyce SD, Zheng X, Maeda N, Kuroda $\mathrm{T}$, Ide C. Gender and age-related differences in corneal topography. Cornea 200120 (3) 270-6.

23. Young G, Allsopp G, Inglis A, Watson S. Comparative performance of disposable soft contact lenses. Cont Lens Ant Eye 199720 (1) 13-21.

24. Maldonado CM, Efron N. Impact of manufacturing technology and material composition on the clinical performance of hydrogel lenses. Optom Vis Sci 200481 (6) 442-54.

25. White $\mathrm{P}, \mathrm{Cho} \mathrm{P}$. Legal issues in contact lens practice with special reference to the practice of orthokeratology. Ophthal Physiol Opt 200323 (2) 151-161.

26. Bennett ES, Weissman BA. Clinical Contact Lens Practice. Philadelphia: JB Lippincott Company 1990 pg 864.
27. Collins MJ, Carney LG. Compliance with care and maintenance procedures amongst contact lens wearers. Clin and Exp Optom 198669 (5) 174-177.

28. Steinemann TL, Fletcher M, Bonny A, Harvey RA, Hamlin D, Zloty P, Besson M, Walter K, Gagnon M. Over-the-counter decorative contact lenses: cosmetic or medical devices? A case series. Eye Contact Lens 200531 194-200.

29. Steinemann TL, Pinninti U, Szczotka LB, Eiferman RA, Price FW Jr. Ocular complications associated with the use of cosmetic contact lenses from unlicensed vendors. Eye Contact Lens 200329 196-200.

30. Ang G S, Goldsmith C, Shenoy R, Astbury N, Burton R. 'Football' contact lenses - an own goal. J R Soc Med 2004 97 (9) 437-438.

31. Statistics South Africa. Statistical release P0302 mid-year population estimates 2009. http://www.statssa.gov.za/PublicationsHTML/p03022009/html/p03022009.html (last accessed 26/10/09). 